\title{
Fluorescence properties of nanoaggregates of pyrene ammonium derivative and its photoinduced dissolution and reaction in tetrahydrofuran/aqueous solutions
}

\author{
Fuyuki Ito*, Takehiro Sagawa, and Hirotaka Koshiyama \\ Department of Chemistry, Faculty of Education, Shinshu University, 6-ro \\ Nishinagano, Nagano 380-8544, Japan
}

Corresponding author footnote

Tel: +81-26-238-4114; Fax: +81-26-238-4114; E-mail: fito@shinshu-u.ac.jp

Abstract

\begin{abstract}
We studied the fluorescence properties of nanoaggregates of pyrene ammonium derivative (PyAm) and their photoinduced dissolution and reaction in tetrahydrofuran (THF)/aqueous solution. The final concentration (dye concentration after reprecipitation) dependence of the fluorescence peak was measured. The fluorescence peak of PyAm that originated from the excimer is shifted to the long wavelength side increasing with the final concentration, which is ascribable to the characteristic fluorescence spectral changes depending on their size. The size-dependent fluorescence change in the nanoaggregates is related to some molecular conformation, packing, and elastic properties of the nanoparticles at the surface. To understand the fluorescence properties of the intermediates from aggregates to crystals is important for the studies of organic nanocrystals/aggregates prepared by the reprecipitation methods. We also determined the fluorescence spectra of the PyAm nanoaggregates in a THF/aqueous solution by photoinduced dissolution and reaction. It originated from the photochemical reaction between PyAm and THF. Changes in the perpendicular light scattering intensity by photoirradiation supports the photoinduced dissolution of PyAm in the THF/aqueous solution. These findings are important in the research field of photochemical reactions in organic nanocrystals.
\end{abstract}

\section{Keywords}

Organic nanocrystal; fluorescence properties; size dependence; photochemical reaction; photoinduced dissolution 


\section{Introduction}

Organic nano-materials are widely investigated in terms of their unique optical properties [1]. Kasai and Nakanishi et al. first reported that the fluorescence spectra of perylene nanocrystals, which were prepared by the reprecipitation method, depended on their size, and the fluorescence spectra of the nanoparticles differ from that of the bulk crystals [2,3]. There are many reports about organic nanoparticles in the past decade [4-7]. They proposed lattice softening of the nanoparticles as a cause of the size-dependent optical properties. Yao et al. reported the size dependence of the emission spectra for pyrazoline nanoparticles, which originated from the formation of charge transfer excitons due to the flexibility and various inter- and intramolecular overlapping structures in the nanoparticles [5].

We have investigated the fluorescence properties of pyrene ammonium derivative (PyAm, Figure 1) in poly(vinylalcohol) (PVA) thin films [8]. The fluorescence spectra of PyAm in the PVA film at a lower concentration showed only a monomer emission. The fluorescence peak shifted to the long wavelength side with the increasing dye concentration, which was assigned to a dimer or excimer-like emission. The concentration-dependent fluorescence changes originated from the PyAm aggregates formed in the PVA matrix by phase separation. The size of the PyAm aggregates in the PVA thin films increased with the increasing dye concentration. The peaks of the dimer and/or excimer-like fluorescence originated from the aggregates that shifted to the long wavelength side with the increasing concentration. This was ascribable to the size effect due to stabilization of the surface free energy. Changes in the fluorescence spectra were related to the formation of nanocrystals and/or aggregates in the films by phase separation, which was presumed to show a hierarchal change (like a consecutive reaction) of the fluorescence property depending on the domain size. We have proposed that the concentration-dependent fluorescence spectral change is due to matrix isolation and/or freezing of the crystal nuclei growth by the polymer chain. In addition, we have also reported similar phenomena and findings for the perylene ammonium derivative (PeryAm) [9].<smiles>C[N+](C)(C)CC(=O)c1ccc2ccc3cccc4ccc1c2c34</smiles>

Figure 1 Chemical structure of PyAm.

Studies regarding the organic nanocrystals/aggregates prepared by the reprecipitation methods are useful in order to understand the fluorescence properties of the intermediates from the aggregates to crystals. In this paper, we report the final concentration (dye concentration after reprecipitation) dependence of the fluorescence peak of PyAm nanoaggregates, and we first observed the photoinduced dissolution and reaction for the nanoaggregates of pyrene ammonium derivative in THF. It is important to study the photochemical reaction of an organic nanocrystal as chemical intermediates.

\section{Experimental}

The pyrene ammonium derivative (trimethyl-(2-oxo-2-pyren-1-yl-ethyl)-ammonium bromide; PyAm, Figure 1) was synthesized according to a previous report [10].

Special grade (Kanto Chemical) THF was commercially available and used without further purification. The distilled THF were obtained from Special grade THF using conventional distillation glassware. Ultrapure water $\left(18.2 \mathrm{M} \Omega \cdot \mathrm{cm}^{-1}\right)$ was also used as a dispersion medium.

An aqueous solution of PyAm $\left(1.0 \times 10^{-3} \mathrm{~mol} \cdot \mathrm{dm}^{-3}\right)$ was rapidly injected into vigorously stirred THF (from 0.5 to $5 \mathrm{~mL}$ ) at ambient temperature using a microsyringe to prepare the nanoaggregates. The final concentration of the PyAm solution after the reprecipitation ranged from $4.0 \times 10^{-6} \mathrm{~mol} \cdot \mathrm{dm}^{-3}$ to $1.7 \times 10^{-4} \mathrm{~mol} \cdot \mathrm{dm}^{-3}$. In the photoirradiation experiments, the PyAm aggregates THF/aqueous solution was constant with an injection of $100 \mu \mathrm{L}$ of the PyAm aqueous solution into $2 \mathrm{~mL}$ of THF. The final concentration was $4.8 \times 10^{-5} \mathrm{~mol} \cdot \mathrm{dm}^{-3}$.

The UV-Vis absorption spectra were measured by a Shimadzu UV-2450 spectrometer. The fluorescence spectra were recorded by a Shimadzu RF-5300PC fluorescence spectrophotometer. All experiments were carried out at ambient temperature.

\section{Results and discussion}

\section{Fluorescence properties of PyAm nanoaggregates as a function of the final concentration:}

We measured the fluorescence spectra of the reprecipitated PyAm. Figure $2 \mathrm{a}$ shows the fluorescesnce spectra of PyAm in a THF/aqueous solution after the reprecipitation. For the dilute solution, such as $4.0 \times 10^{-6} \mathrm{~mol} \cdot \mathrm{dm}^{-3}$, fluorescence peaks were observed at 390,412 and $433 \mathrm{~nm}$. With the increasing final concentration, the peaks shifted to the long wavelength side. For the 
$1.7 \times 10^{-4} \mathrm{~mol} \cdot \mathrm{dm}^{-3}$ concentration, only one broad peak observed at $469 \mathrm{~nm}$. The peak at $390 \mathrm{~nm}$ is originated from Raman scattering from THF, and that at $412 \mathrm{~nm}$ originated from the small amount of impurity or photoproduct described below (even in the distilled one). The peaks of the PyAm fluorescence at 433 and $469 \mathrm{~nm}$ are assignable to the excimer fluorescence that changed depending on the final concentration. Table.1 summarized the relation between solvent $\left(\mathrm{H}_{2} \mathrm{O} / \mathrm{THF}\right)$ ratio and the PyAm fluorescence peak. The fluorescence peak shifted to the long wavelength side with increasing the water contents. The fluorescence peaks depends on not only the final concentration, but also the ratio of $\mathrm{H}_{2} \mathrm{O}$ /THF. These findings indicate that the aggregation formation depends on the solvent contents (that is solubility), which most probably results in the size changes of PyAm nanoaggregates. The fluorescence spectra of the aggregates, however, differ from that of the powder. This is means that the PyAm bulk crystal was not generated by the present reprecipitation condition. As an example, the SEM photograph of the PyAm nanoaggregates was obtained by adding $0.5 \mathrm{~mL}$ of the PyAm aqueous solution to $0.5 \mathrm{~mL}$ of THF, which shows the ball-like aggregate with a diameter of $450 \mathrm{~nm}$ as shown in Figure 3.
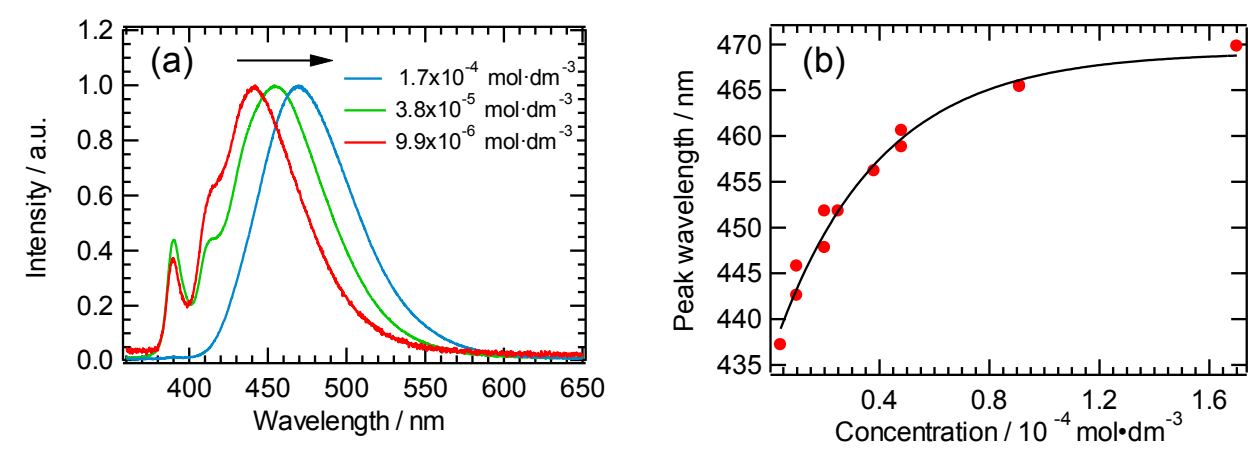

Figure 2 (a) Final concentration dependence of fluorescence spectra of PyAm aggregates in THF/aqueous solution excited at $\lambda=350 \mathrm{~nm}$. (b) Peak wavelength of PyAm aggregates as a function of the concentration. The solid line is a visual guide.

Table 1 The relation between the PyAm fluorescence peaks and the ratio of water and THF.

\begin{tabular}{cccc}
\hline & \multicolumn{3}{c}{ Peak wavelength $/ \mathrm{nm}$} \\
$\mathrm{THF} / \mathrm{mL}$ & 20 & 50 & 100 \\
\hline 0.5 & 454.0 & 465.6 & 469.4 \\
1.0 & 445.6 & 459.8 & 465.0 \\
2.0 & 444.2 & 450.0 & 458.2 \\
5.0 & 432.8 & 442.0 & 447.8 \\
\hline
\end{tabular}

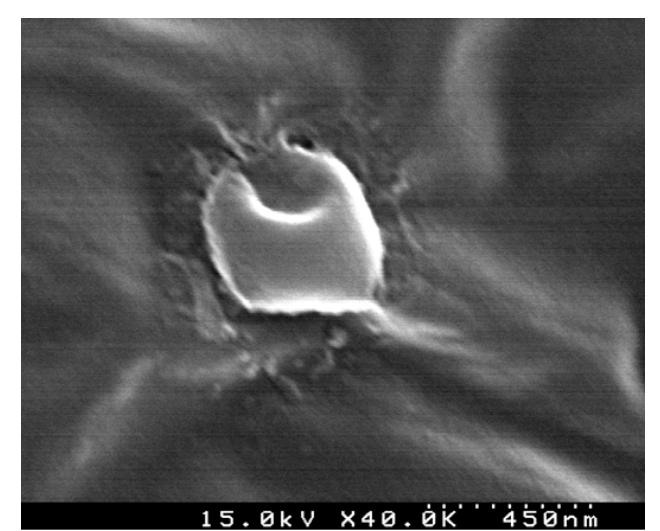

Figure 3 SEM photograph of PyAm nano aggregates obtained by adding $0.5 \mathrm{~mL}$ of the PyAm aqueous solution to $0.5 \mathrm{~mL}$ of THF.

The final concentration dependence of the peaks of the PyAm fluorescence is shown in Figure 2b. The fluorescence peak of PyAm originated from the excimer is shift to the long wavelength side by increasing the final concentration, which is ascribable to the characteristic 
fluorescence spectral changes depending on the particle size. It has been widely reported for organic nanoparticles. The fluorescence spectra of the nanoparticles differ from that of the bulk crystals. Kasai et al. proposed lattice softening in the nanoparticles as a cause of the sizedependent optical properties [2,3]. Asahi et al. developed and proposed a model of the sizedependent optical properties of perylene nanoparticles [10]. In order to explain the observed fluorescence spectral change, we adapted a model concerning the size-dependent optical properties of perylene nanoparticles. Figure 4 is a schematic representation of the potential surface of the monomer and excimer states. The abscissa denotes the relative displacement of the excimer state from the bottom of the ground state. The ground and excited Franck-Condon states were assumed to have the same potential to the bulk crystal. It is well-known that electronic excitation energy is trapped in a sandwich-like pair of two molecules, i.e., excimer formation, in the crystals [12]. Because the monomer fluorescence peak of the nanocrystal is identical to that of the bulk one, half the value of the free exciton bandwidth can be considered to be independent of size. Therefore, the size dependences of the excimer fluorescence peak should be attributed to reduction in the lattice relaxation energy $\left(E_{\mathrm{LR}}\right)$ with the decreasing size. The size dependence of $E_{\mathrm{LR}}$ is attributable to a change in the elastic properties due to excimer formation of the nanocrystal with the size. The lattice instability caused by the large surface-to-volume ratio is most likely responsible for lowering the lattice vibration or rigidity with the decreasing crystal size, which leads to the upshift of the excimer energy level as illustrated in Figure 4. It is proposed that the energy difference between the excimer and ground states monotonously increases with the surface-to-volume ratio. For example, it is reported that the melting temperature of metallic nanoparticles and some organic nanocrystals or aggregates is lower than that of the bulk. The increase in the free energy of the nanocrystal or aggregate results in a contribution of the surface free energy. The increase in free energy of the nanocrystal or aggregate most probably results in a contribution of the surface free energy, because the size effect on the organic nanoparticles is related to some molecular conformation, packing, and elastic properties of the nanoparticles at the surface. These findings indicate that the fluorescence spectral change allows us to change the size of the aggregates and crystals, that is, intermediate state.

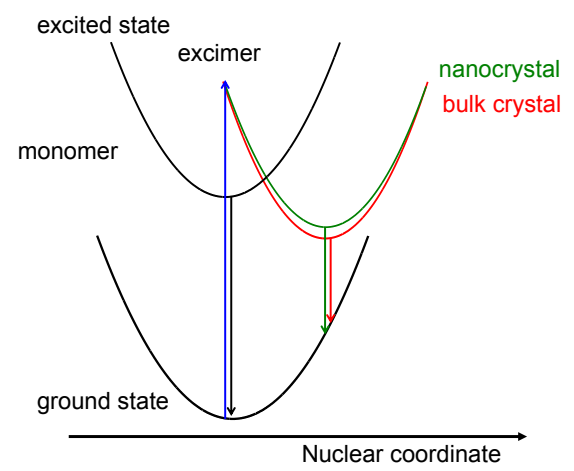

Figure 4 Schematic representation of potential energy surfaces of nano- and bulk crystals.

\section{Fluorescence spectral change of PyAm aggregates by photo-irradiation:}

We are the first to observe the fluorescence spectral change in the PyAm aggregates depending on the photoirradiation. Although THF is widely used as a solvent for reprecipitation methods, trace impurities or photoproduct will exist as mentioned above. Thus, it is very important to study the photophysical properties of the re-precipitated solution, especially for the materials with a low fluorescence quantum yield.

We used a special grade of THF as the solvent for dissolution of the PyAm. Figure 5a shows the changes in the fluorescence spectra of the PyAm aggregates in the special grade THF/aqueous solution with $\lambda=350 \mathrm{~nm}$ as a function of time. Only a broad peak around $456 \mathrm{~nm}$ was observed just after the irradiation, which is assigned to the excimer fluorescence of PyAm as mentioned above. With time, the fluorescence band at $410 \mathrm{~nm}$ appeared and the intensity up to 450 $\mathrm{nm}$ slightly decreased. The band at $390 \mathrm{~nm}$ is identical to the Raman scattering of THF, whose increment can be interpreted as increasing of the tail intensity of the $410 \mathrm{~nm}$ fluorescence band. To check the effect of the stabilizer such as dibutylhydroxytoluene, we performed a distillation of the special grade THF just after the sample preparation. Figure $5 \mathrm{~b}$ shows the changes in the fluorescence spectra. The spectral change behavior in the distilled THF/aqueous solution is identical to that in the special grade THF/aqueous solution, which indicated that the generated fluorescent species did not originate from the stabilizer or small amount of impurities. The excitation spectra of the PyAm reprecipitated solution also changed as a function of time, which indicated that the ground state species changed by the photoirradiation. 

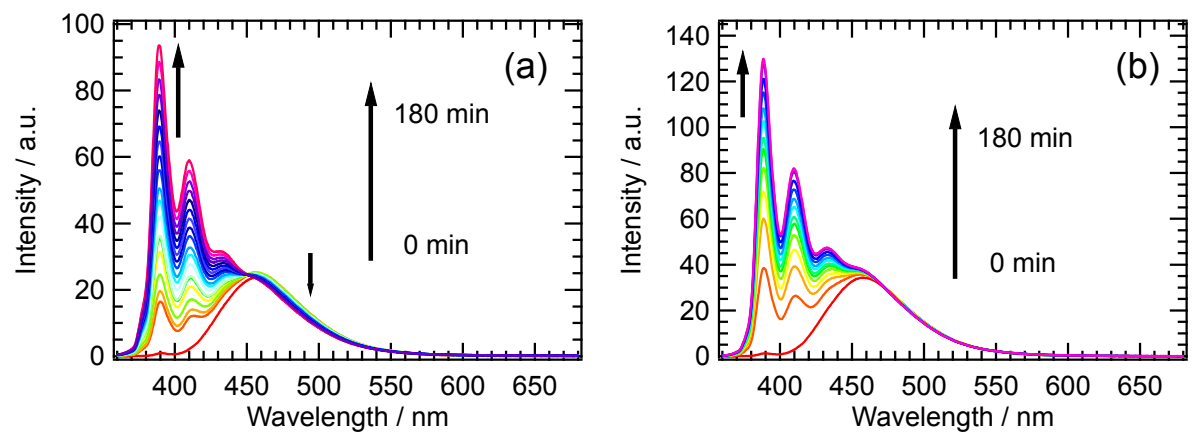

Figure 5 Changes in fluorescence spectra of reprecipitated PyAm in THF/aqueous solution excited at $\lambda=350 \mathrm{~nm}$ as a function of time. THF was of (a) special grade and (b) distilled.

In order to clarify the fluorescence change in the present system, we measured the fluorescence spectra of the PyAm aqueous solution and THF solvent by the photoiraddiation. Figure6a shows the fluorescence spectra of the PyAm aqueous solution as a function of time. These spectra remain unaltered with time, suggesting that the water did not affect the PyAm fluorescence changes. PyAm is also slightly dissolved in THF (less than $1.0 \times 10^{-7} \mathrm{~mol} \cdot \mathrm{dm}^{-3}$ ). We prepared PyAm in a THF solution by filtration of the suspension after an 18-h stand. The fluorescence spectral changes in the PyAm in the THF solution are shown in Figure 5b. The sharp fluorescence bands at 390, 410 and $430 \mathrm{~nm}$ were observed from just after the preparation and monotonically increased with time. These bands correspond to the fluorescence in the THF/aqueous solutions. Figures $6 \mathrm{c}$ and d show the absorption spectra of PyAm in THF before and after a 3-h photoirradiation at $350 \mathrm{~nm}$, and the difference spectrum from after to the before irradiation, respectively. The absorption spectra of PyAm indicate the peak at $365 \mathrm{~nm}$ and shoulder at $390 \mathrm{~nm}$ that originated from PyAm. After the 3-h photoirradiation, the intensity of these bands decreased with the increasing wavelength range from 300 to $350 \mathrm{~nm}$. This finding indicates that the new fluorescence bands are most probably from a photoproduct between PyAm and THF except for the $390 \mathrm{~nm}$ peak (Raman Scattering), ascribed to the excitation light employed for the fluorescence measurements. Unfortunately, the product was not detected by other spectroscopies such as NMR, because the product was not formed in a sufficient concentration for their measurements. The fluorescence quantum yield of the product is probably strong.
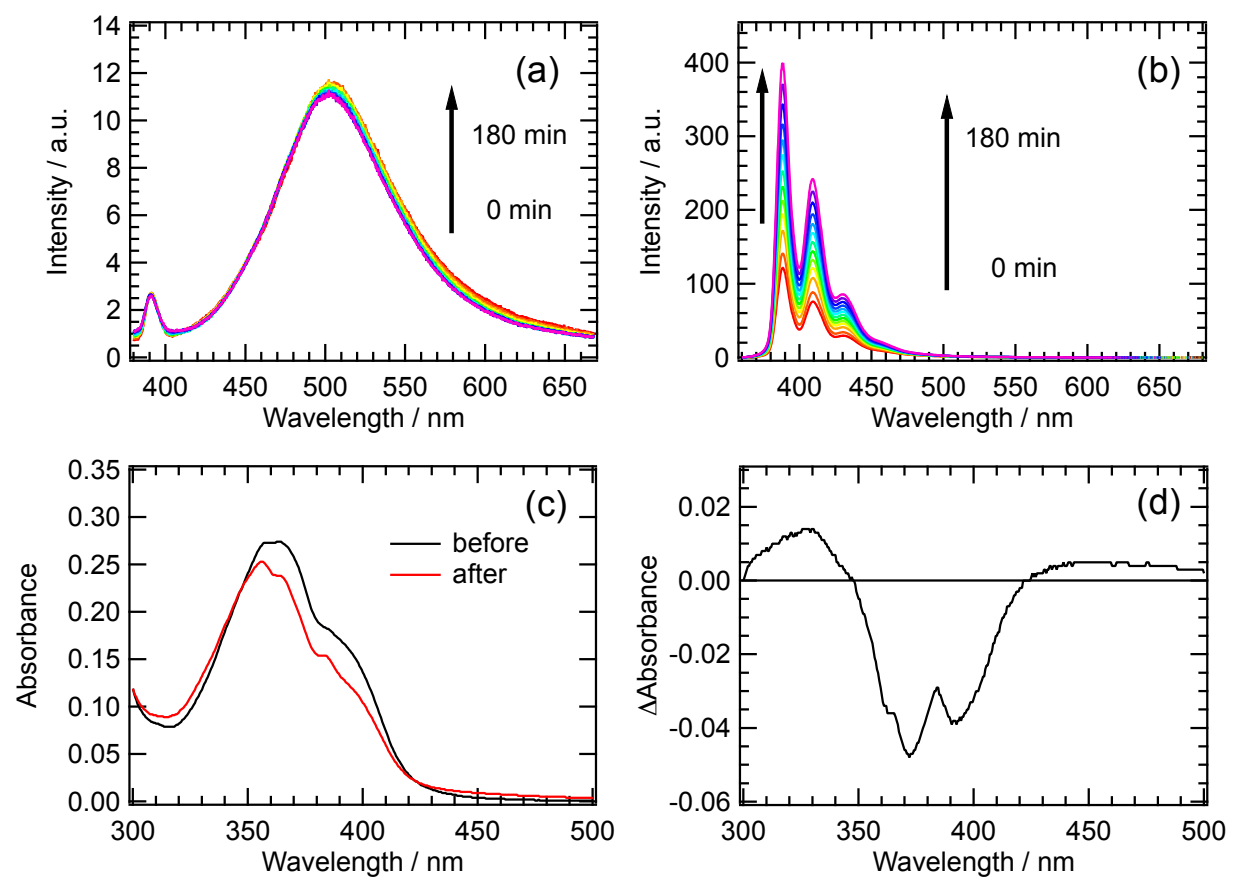

Figure 6 Changes in fluorescence spectra of PyAm (a) in aqueous solution and (b) in THF solution. (c) Absorption spectra of PyAm in THF before and after the photoirradiation and (d) its differential spectrum.

In order to confirm that the spectral change is ascribed to the photochemical reaction, the photoirradiation time dependence was measured. The fluorescence spectral measurements of the reprecipitated PyAm in the THF/aqueous solution were performed under continuous 
photoirradiation and only the spectral scan time. Figure 7 shows the changes in the fluorescence spectra of the PyAm aggregates in the THF/aqueous solution with $\lambda=350 \mathrm{~nm}$ as a function of time under photoirradiation at only the spectral scan time (a) and all through the time course (b). The wavelength scan takes $90 \mathrm{~s}$ for each measurement, and the number of measurements is 45 , therefore, the total irradiation time is estimated to be $67 \mathrm{~min}$. The irradiation time of the during measurement is about 2.7 times greater than that of only the spectral scan time, which corresponds to the fluorescence intensity ratio between them at $435 \mathrm{~nm}$ after $180 \mathrm{~min}$ (about 2.6). These findings indicated that the amount of generated products is proportional to the photoirradiation time, leading to the conclusion that the fluorescence spectral change of the PyAm aggregates will be ascribed to the photochemical reaction between PyAm and THF in solution.
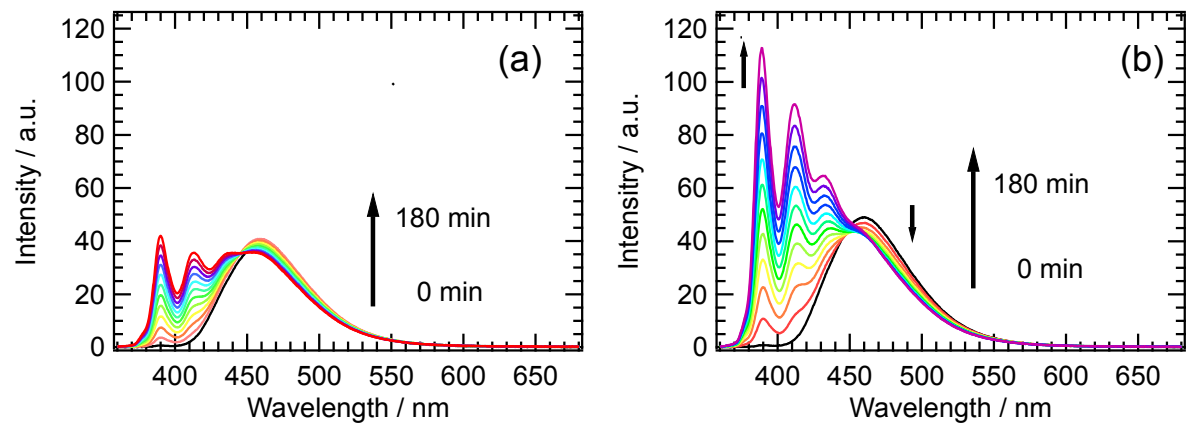

Figure 7 Fluorescence spectra of fluorescence spectra of PyAm aggregates in THF/aqueous solution excited with $\lambda=350 \mathrm{~nm}$ as a function of time during the photoirradiation at only the spectral scan time (a) and at continuously within the time-course measurements (b).

\section{Photoinduced dissolution kinetics of PyAm nano aggregates probed by light scattering intensity:}

We have described that fluorescence spectral change in the PyAm nanoaggregates in a THF/aqueous solution is ascribed to the photochemical reaction between PyAm and THF as the solvent. The slight dissolution of PyAm in THF will influence the reaction. The dissolution behavior of the PyAm nanoaggregates was examined by the time evolution of the perpendicular light scattering intensity by a conventional fluorescence spectrometer. Figure 8 shows the changes in the perpendicular light scattering intensity at $\lambda=350$ and $700 \mathrm{~nm}$ of the PyAm nanoaggregates irradiated at $\lambda=350 \mathrm{~nm}$, which was normalized just after the measurement $(t=0)$. Based on the Rayleigh scattering equation, the scattering intensity $(I)$ can be expressed by the following relation:

$$
I \propto \frac{d^{6}}{\lambda^{4}} c
$$

where $\lambda, d$ and $c$ correspond to the incident light wavelength, particle radius and concentration, respectively. Thus, the decreasing scattering light intensity can be ascribed to the concentration (number of particles) or radius. The offset values are about 0.87 and 0.80 observed at 700 and 350 $\mathrm{nm}$, respectively, which suggestes that the photoinduced dissolution was completely finished within $50 \mathrm{~min}$, although the fluorescence spectral change remained. Decreasing of the scattering intensity was reproduced by the single exponential decay with the time constants of 16 min and $6.1 \mathrm{~min}$ at 700 and $350 \mathrm{~nm}$, respectively. The irradiation wavelength dependence may be the difference in the scattering particle size. The scattering intensity change originated from the photoinduced dissolution phenomena of the PyAm nanoaggregates. Plenary measurements of the mass spectra for PyAm before and after the photoirradiation indicated that no assignable species was generated by the photoirradiation. Based on these experimental findings, we concluded that the photoinduced dissolution is due to the photochemical reaction between PyAm and THF. 


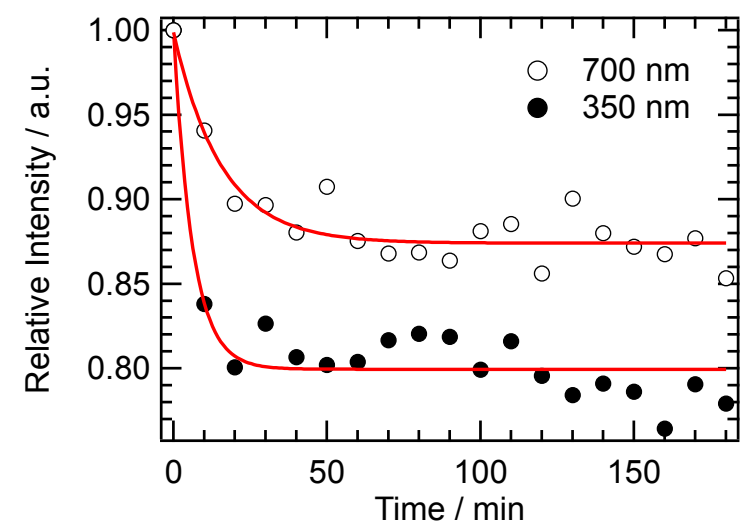

Figure 8 Changes in perpendicular light scattering intensity of PyAm nanoaggregates observed at $\lambda=350$ and $700 \mathrm{~nm}$ by $\lambda=350 \mathrm{~nm}$ photoirradiation.

\section{Conclusion}

In this investigation, we studied the fluorescence spectra of the PyAm nanoaggregates in a THF/aqueous solution for the final concentration dependence of the fluorescence peak and its photoinduced dissolution. The increasing final concentration showed the peaks shift to the long wavelength side, suggesting that the fluorescence spectral change is due to the size change of the aggregates and crystals, that is the intermediate state. We also investigated the dissolution of PyAm nanoaggregates in THF, which originated from the photochemical reaction between PyAm and THF. This phenomenon was confirmed by changes in the perpendicular light scattering intensity. These findings are important for the research field of photochemical reactions in organic nanocrystals.

\section{Acknowledgements}

This work partly supported by a Grant-in-Aid for Young Scientist (B) (No. 21750021) from the Ministry of Education, Culture, Sports, Science, and Technology (MEXT) of the Japanese Government, by the Iketani Science and Technology Foundation, and by a Grant-in-Aid for Young Scientist of Exploratory Research from Shinshu University.

\section{References}

[1] H. Masuhara, H. Nakanishi, K. Sasaki, Eds., Single Organic Nanoparticles (Springer, Berlin, 2003).

[2] H. Kasai, H. S. Nalwa, H. Oikawa, S. Okada, H. Matsuda, N. Minami, A. Kakuda, K. Ono, A. Mukoh, H. Nakanishi, Jpn. J. Appl. Phys. 31, L1132 (1992).

[3] H. Kasai, H. Kamatani, Y. Yoshikawa, S. Okada, H. Oikawa, A. Watanabe, O. Itoh, H.

Nakanishi, Chem. Lett. 1181 (1997).

[4] U. Rohr, P. Schlichting, A. Bohm, M. Gross, K. Meerholz, C. Brauchle, K. Mullen, Angew. Chem. Int. Ed. 37, 1434 (1998).

[5] D. B. Xiao, X. Lu, W. S. Yang, H. B. Fu, Z. G. Shuai, Y. Fang, J. N. Yao, J. Am. Chem. Soc. 125, 6740 (2003).

[6] B. K. An, S. K. Kwon, S. D. Jung, S. Y. Park, J. Am. Chem. Soc. 124, 14410 (2002).

[7] H. B. Fu, J. N. Yao, J. Am. Chem. Soc. 123, 1434 (2001).

[8] F. Ito, T. Kakiuchi, T. Sakano, T. Nagamura, Phys. Chem. Chem. Phys. 12, 10923 (2010).

[9] F. Ito, Y. Ugachi, T. Sasaki, Chem. Lett. 41, 282 (2012).

[10] N. Nakashima, Y. Tanaka, Y. Tomonari, H. Murakami, H. Kataura, T. Sakaue, K.

Yoshikawa, J. Phys. Chem. B 109, 13076 (2005).

[11] T. Asahi, T. Sugiyama, H. Masuhara, Acc. Chem. Res. 41, 1790 (2008).

[12] Y. Toyozawa, Pure Appl. Chem. 69, 1171 (1997). 